PROCEEDINGS OF THE

AMERICAN MATHEMATICAL SOCIETY

Volume 136, Number 6, June 2008, Pages 2155-2163

S 0002-9939(08)09336-2

Article electronically published on January 3, 2008

\title{
NON-GAUSSIAN UPPER ESTIMATES FOR HEAT KERNELS ON SPACES OF HOMOGENEOUS TYPE
}

\author{
DACHUN YANG AND YUAN ZHOU
}

(Communicated by Hart F. Smith)

\begin{abstract}
The authors extend non-Gaussian upper estimates on the positive real axis to a certain sector of $\mathbb{C}$ including the positive real axis for heat kernels on spaces of homogeneous type, which are known to be holomorphic in that sector.
\end{abstract}

\section{INTRODUCTION}

The study on the behavior of heat kernels has long been an active topic in functional analysis, partial differential equations and harmonic analysis. It is known that heat kernel bounds such as Gaussian bounds or Poisson bounds imply various useful properties of operators such as $L^{p}$ spectral invariance [1, 7], $L^{p}$-boundedness of Riesz means [3], bounded holomorphic functional calculi on $L^{p}$ spaces [10] or $L^{p}$ analyticity of the corresponding semigroup 13. Recently, by using certain upper estimates and the time derivative upper estimates for heat kernels, Auscher, Duong and McIntosh [2, and Duong and Yan 11, 12] introduced some Hardy spaces and their dual spaces associated with certain differential operators, and established bounded holomorphic functional calculi on such Hardy spaces.

The extension of Gaussian upper bounds for heat kernels on $t>0$ to complex time $z \in \Sigma_{\pi / 2}=\{z \in \mathbb{C}: \Re(z)>0\}$ was first given by Davies; see Theorem 3.4.8 in 6]. Here and in what follows, we use $\Re(z)$ to denote the real part of complex number $z$. Carron, Coulhon and Ouhabaz generalized this result to the heat kernel which satisfies the Gaussian upper bounds of order $m \geq 2(m=2$ in [6. Theorem 3.4.8]), and to the space of homogeneous type in the sense of Coifman and Weiss (4]) and its open subset; see Proposition 4.1 in [3] and also Theorem 7.2 in [14. The fact that this type of Gaussian upper estimate holds for all $z \in \Sigma_{\pi / 2}$ plays a central role in establishing the $L^{p}$ spectral invariance of the generator and the $L^{p}$-boundedness of Riesz means; see [3, 6]. On the other hand, Davies established a general criteria to estimate the time derivative of the heat kernel on the quasi-metric measure space which may not satisfy the doubling condition, where the heat kernel is not deliberately assumed to satisfy any type of Gaussian upper bounds; see Theorem 4

Received by the editors April 20, 2007.

2000 Mathematics Subject Classification. Primary 47D60; Secondary 58J35.

Key words and phrases. Heat kernel, semigroup, time derivative, non-Gaussian upper estimate, space of homogeneous type.

The first author was supported by the National Science Foundation for Distinguished Young Scholars (No. 10425106) and NCET (No. 04-0142) of the Ministry of Education of China.

(C)2008 American Mathematical Society

Reverts to public domain 28 years from publication 
in 8]. Moreover, from his proof, one can deduce the upper estimate for the heat kernel on complex time $z \in \Sigma_{\psi}=\{z \in \mathbb{C}:|\arg z|<\psi\}$ with $\tan \psi \leq 1 / 8$; see also Proposition 1 below. However, to extend the bounded holomorphic functional calculus on $L^{2}$ to $L^{p}$ with $p \in(1, \infty)$ for an analytic semigroup whose kernels are known to satisfy certain upper bounds only for all $t>0$, one first needs to verify these kernels having uniform upper estimates in a sector which is strictly contained in $\Sigma_{\pi / 2}$ and may strictly contain $\Sigma_{\psi}$; see Proposition 3.3 and the proof of Theorem 3.3 in [10. Moreover, Proposition 3.3 in 10 was used in 10, 5, 9, and cited in [12, 3, 11. However, it seems that there is a gap in the proof of Proposition 3.3 in [10. In this paper, we will seal this gap by establishing its more general version.

To be precise, we extend the upper bound on $t>0$ to the complex time $z \in \Sigma_{\phi}$ with $\phi \in(0, \pi / 2]$ for heat kernels, which satisfy certain decay conditions on a certain space of homogeneous type, and then establish the time derivative estimate of heat kernels; see Theorem 1 and Theorem 2 below. To compare the results here with the corresponding results of Davies in [8], we also state a slightly more general version of Theorem 4 in [8]; see Proposition 1 below. Applying Proposition 1, we obtain the upper estimates for complex time $z \in \Sigma_{\psi}$ with $\tan \psi \leq 1 / 8$ and a derivative of certain heat kernels.

We finally make some conventions. We always use $C_{i}$ with $i \in \mathbb{N}$ to denote a positive constant that is independent of the main parameters involved but whose value does not change in different occurrences.

\section{Main Results}

Let $(\mathcal{X}, d, \mu)$ be a space of homogeneous type in the sense of Coifman and Weiss [4. Namely, $\mathcal{X}$ is a set, $d$ is a quasi-metric on $\mathcal{X}$ and $\mu$ is a positive Borel regular measure satisfying the fact that there exists a constant $C_{1}>0$ such that for all $x \in \mathcal{X}$ and $r>0$,

$$
V(x, 2 r) \leq C_{1} V(x, r),
$$

and where and in what follows, $B(x, r)=\{y \in \mathcal{X}: d(x, y)<r\}$ and $V(x, r)=$ $\mu(B(x, r))$. From (1), it follows that there exist constants $n>0$ and $C_{2}>0$ such that for all $\lambda>1, x \in \mathcal{X}$ and $r>0$,

$$
V(x, \lambda r) \leq C_{2} \lambda^{n} V(x, r) .
$$

For $\phi \in(0, \pi / 2]$, set

$$
\Sigma_{\phi}=\{z \in \mathbb{C}: z \neq 0 \text { and }|\arg z|<\phi\} .
$$

Let $m>0$. For $z \in \Sigma_{\phi}$ and $\lambda \in(0, \infty)$, let

$$
f(z, \lambda)=C_{2}\left(\frac{\lambda}{z}\right)^{n / m} e^{z / \lambda}
$$

Then for each $\lambda \in(0, \infty),[f(\cdot, \lambda)]^{-1}$ is holomorphic in $\Sigma_{\phi}$, and for all $\lambda \in(0, \infty)$ and $z \in \Sigma_{\psi}$,

$$
\frac{|f(z, \lambda)|}{|f(\Re(z), \lambda)|}=\frac{1}{C_{2}}|f(z, \Re(z))|=(\cos \arg z)^{n / m} .
$$

Furthermore, the following useful Lemma 1 was established in the proof of Theorem 7.2 in 14 .

Lemma 1. For all $t, \lambda>0$ and $x \in \mathcal{X}, V\left(x, \lambda^{1 / m}\right) \leq f(t, \lambda) V\left(x, t^{1 / m}\right)$. 
We now turn to the main result of this paper. Some ideas come from Theorem 4 in [8] of Davies and also Proposition 3.3 in [10].

Theorem 1. Let $\phi \in(0, \pi / 2]$, and $z \in \Sigma_{\phi} \rightarrow K_{z} \in \mathbb{C}$ be the kernel of a holomorphic family of bounded operators on $L^{2}(\mathcal{X})$. Assume that there exist $m>0, \kappa>0$ and $C_{3} \geq 1$ such that

(i) for all $x, y \in \mathcal{X}$ and $z \in \Sigma_{\phi}$,

$$
\left|K_{z}(x, y)\right| \leq C_{3} \frac{1}{V\left(x,[\Re(z)]^{1 / m}\right)}
$$

(ii) there exists a bounded function $b$ on $[0, \infty)$ and a constant $C_{4}>0$ satisfying that for all $s, \tau \in[0, \infty)$,

$$
b(s) \leq C_{3} b(\tau)\left(\frac{1+\tau}{1+s}\right)^{\kappa} \exp \left(C_{4} \frac{1+s}{1+\tau}\right)
$$

such that for all $x, y \in \mathcal{X}$,

$$
\left|K_{t}(x, y)\right| \leq C_{3} \frac{1}{V\left(x, t^{1 / m}\right)} b\left(d^{m}(x, y) t^{-1}\right) .
$$

Then for each $\epsilon \in(0,1], \psi \in(0, \phi)$ and $\theta \in(0, \epsilon \psi)$, there exists a constant $C_{5}>0$ such that for all $x, y \in \mathcal{X}$ and $z \in \Sigma_{\theta}$,

$$
\left|K_{z}(x, y)\right| \leq C_{5} \frac{(\cos \arg z)^{n / m+\kappa}}{(\cos \psi)^{n / m+\kappa}} \frac{1}{V\left(x,[\Re(z)]^{1 / m}\right)}\left[b\left(d^{m}(x, y) \Re\left(z^{-1}\right)\right)\right]^{1-\epsilon} .
$$

Proof. For $z \in \Sigma_{\phi}$ and $\tau \in(0, \infty)$, let

$$
g(z, \tau)=C_{3}\left(\frac{1+\tau}{1+z}\right)^{\kappa} \exp \left(C_{4} \frac{1+z}{1+\tau}\right) .
$$

Then (5) tells us that $b(s) \leq g(s, \tau) b(\tau)$ for all $s, \tau \in(0, \infty)$. It is also easy to see that for each $\tau \in(0, \infty),[g(\cdot, \tau)]^{-1}$ is holomorphic on $\Sigma_{\phi}$. Applying the Taylor expansion for the exponential function, we then obtain that for all $s, \tau \in(0, \infty)$,

$$
g(s, \tau) \geq \frac{C_{3}}{\lfloor\kappa+1\rfloor !}
$$

where $\lfloor\kappa+1\rfloor$ denotes the minimal integer no more than $\kappa+1$. Moreover, for all $z \in \Sigma_{\phi}$ and $\tau \in(0, \infty)$

$$
\frac{1}{2^{\kappa}}(\cos \arg z)^{\kappa} \leq|g(z, \Re(z))|=\frac{|g(z, \tau)|}{|g(\Re(z), \tau)|}=\left|\frac{1+\Re(z)}{1+z}\right|^{\kappa} \leq \frac{2^{\kappa}}{(\cos \arg z)^{\kappa}} .
$$

To verify (9), it suffices to verify that for all $z \in \Sigma_{\pi / 2}$,

$$
\frac{1}{2} \cos \arg z \leq\left|\frac{1+z}{1+\Re(z)}\right| \leq \frac{2}{\cos \arg z} .
$$

In fact, when $\Re(z)>1$, then

$$
\frac{1}{2} \cos \arg z \leq\left|\frac{z}{2 \Re(z)}\right| \leq\left|\frac{1+z}{1+\Re(z)}\right|<\left|\frac{1+z}{\Re(z)}\right|<2\left|\frac{z}{\Re(z)}\right|=2 \frac{1}{\cos \arg z},
$$

while when $\Re(z) \leq 1$, then

$$
|z|=\frac{\Re(z)}{\cos \arg z} \leq \frac{1}{\cos \arg z}
$$


which also leads to the fact that

$$
\frac{1}{2} \cos \arg z \leq \frac{1}{2}<\left|\frac{1+z}{1+\Re(z)}\right|<1+|z| \leq 1+\frac{1}{\cos \arg z} \leq 2 \frac{1}{\cos \arg z} .
$$

Thus, (9) holds.

Now fix $\lambda, \tau>0$. For $z \in \Sigma_{\phi}$ and $x, y \in \mathcal{X}$, set

$$
F_{\lambda, \tau}(z)=K_{z}(x, y) V\left(x, \lambda^{1 / m}\right)[f(z, \lambda)]^{-1}\left[g\left(d^{m}(x, y) z^{-1}, \tau\right)\right]^{-1} .
$$

Since $[f(z, \lambda)]^{-1},\left[g\left(d^{m}(x, y) z^{-1}, \tau\right)\right]^{-1}$ and $K_{z}(x, y)$ are holomorphic on $\Sigma_{\phi}, F_{\lambda, \tau}(z)$ is also holomorphic on $\Sigma_{\phi}$. Moreover, for $t \in(0, \infty)$, by (5), (6) and Lemma 1 we have

$$
\begin{aligned}
\left|F_{\lambda, \tau}(t)\right| & \leq\left|K_{t}(x, y)\right| V\left(x, \lambda^{1 / m}\right)|f(t, \lambda)|^{-1}\left|g\left(d^{m}(x, y) t^{-1}, \tau\right)\right|^{-1} \\
& \leq C_{3} \frac{V\left(x, \lambda^{1 / m}\right)}{V\left(x, t^{1 / m}\right)}|f(t, \lambda)|^{-1} b\left(d^{m}(x, y) t^{-1}\right)\left|g\left(d^{m}(x, y) t^{-1}, \tau\right)\right|^{-1} \\
& \leq C_{3} b(\tau)
\end{aligned}
$$

For any $\psi \in(0, \phi)$ and $z \in \Sigma_{\psi}$, by Lemma 1, (3), (4), (8) and (9), we obtain

$$
\begin{aligned}
\left|F_{\lambda, \tau}(z)\right| & \leq\left|K_{z}(x, y)\right| V\left(x, \lambda^{1 / m}\right)|f(z, \lambda)|^{-1}\left|g\left(d^{m}(x, y) z^{-1}, \tau\right)\right|^{-1} \\
& \leq C_{1} \frac{V\left(x, \lambda^{1 / m}\right)}{V\left(x,[\Re(z)]^{1 / m}\right)}|f(z, \lambda)|^{-1}\left|g\left(d^{m}(x, y) z^{-1}, \tau\right)\right|^{-1} \\
& \leq C_{1} C_{2} \frac{\lfloor\kappa+1\rfloor !|f(\Re(z), \lambda)|}{C_{3}} \frac{\left|g\left(d^{m}(x, y) \Re\left(z^{-1}\right), \tau\right)\right|}{|f(z, \lambda)|} \frac{1}{\left|g\left(d^{m}(x, y) z^{-1}, \tau\right)\right|} \\
& \leq C_{1} C_{2} 2^{\kappa} \frac{\lfloor\kappa+1\rfloor !}{C_{3}} \frac{1}{(\cos \arg z)^{n / m+\kappa}} \\
& \leq C_{1} C_{2} 2^{\kappa} \frac{\lfloor\kappa+1\rfloor !}{C_{3}} \frac{1}{(\cos \psi)^{n / m+\kappa}} .
\end{aligned}
$$

Set $C_{6}=\max \{1, \sup \{b(t): t>0\}\}$ and $C_{5}=C_{1} C_{2}\left(C_{3}\right)^{-1} C_{6} 2^{\kappa}(\lfloor\kappa+1\rfloor !)$. Fix $\delta>0$. Let $\alpha=\psi / \pi, z^{\alpha}=\exp (\alpha \log z)$ for $z \in \mathbb{C}$ with $\arg z \in[-\pi, \pi]$ and

$$
H_{\lambda, \tau, \delta}(z)=C_{5}^{-1}(\cos \psi)^{n / m+\kappa} F_{\lambda, \tau}\left(z^{\alpha}+\delta\right) .
$$

Then $H$ has a cut along the negative real axis but is analytic elsewhere. Moreover, (11) and (12) imply that $\left|H_{\lambda, \tau, \delta}(z)\right| \leq 1$ for $z \in \mathbb{C}$ and $\left|H_{\lambda, \tau, \delta}(t)\right| \leq b(\tau) / C_{6} \leq 1$ for $t>0$. If we define

$$
J_{\lambda, \tau, \delta}(z)=\log \left|H_{\lambda, \tau, \delta}(z)\right|
$$

for $z \in \mathbb{C} \backslash(-\infty, 0]$, then $J$ is harmonic where it is finite, and moreover, $J$ is subharmonic on its domain and takes values in $[-\infty, 0]$. For $u \in \mathbb{R}$ and $v>0$, using the Poisson formula on the half-plane and by $J(s) \leq 0$ for $s \in(-\infty, \infty)$, we 
then have

$$
\begin{aligned}
J(u+i v) & \leq \frac{v}{\pi} \int_{-\infty}^{\infty} \frac{J_{\lambda, \tau, \delta}(s)}{(u-s)^{2}+v^{2}} d s \\
& \leq \frac{v}{\pi} \int_{0}^{\infty} \frac{J_{\lambda, \tau, \delta}(s)}{(u-s)^{2}+v^{2}} d s \\
& \leq \frac{v}{\pi} \int_{0}^{\infty} \frac{\log \left|H_{\lambda, \tau, \delta}(s)\right|}{(u-s)^{2}+v^{2}} d s \\
& \leq\left[\log \left(\frac{b(\tau)}{C_{6}}\right)\right] \frac{v}{\pi} \int_{0}^{\infty} \frac{1}{(u-s)^{2}+v^{2}} d s \\
& \leq \pi^{-1}\left(\pi / 2+\tan ^{-1}(u / v)\right) \log \left(\frac{b(\tau)}{C_{6}}\right) \\
& \leq\left(1-\pi^{-1} \tan ^{-1}(v / u)\right) \log \left(\frac{b(\tau)}{C_{6}}\right) .
\end{aligned}
$$

Thus for $0 \leq \tan ^{-1}(v / u) \leq \epsilon \pi$,

$$
J_{\lambda, \tau, \delta}(u+i v) \leq \log \left(\left[\frac{b(\tau)}{C_{6}}\right]^{1-\epsilon}\right) \leq \log [b(\tau)]^{1-\epsilon},
$$

which still holds for $u \in \mathbb{R}$ and $v<0$ with $0 \leq-\tan ^{-1}(v / u) \leq \epsilon \pi$ by an argument similar to the case $v>0$. From this, we deduce that for $w \in \mathbb{C}$ with $|\arg w| \leq \epsilon \pi$,

$$
\left|H_{\lambda, \tau, \delta}(w)\right| \leq[b(\tau)]^{1-\epsilon} .
$$

For $\theta \in(0, \epsilon \psi)$ and $z \in \Sigma_{\theta}$, choosing $\delta$ small enough such that $z-\delta \in \Sigma_{\theta}$, and letting $w=(z-\delta)^{1 / \alpha}$, we then obtain

$$
\left|F_{\lambda, \tau}(z)\right|=C_{5} \frac{1}{(\cos \psi)^{n / m+\kappa}}\left|H_{\lambda, \tau, \delta}(w)\right| \leq C_{5} \frac{1}{(\cos \psi)^{n / m+\kappa}}[b(\tau)]^{1-\epsilon} .
$$

Further choosing $\lambda=\Re(z)$ and $\tau=d^{m}(x, y) \Re\left(z^{-1}\right)$, from (3), (9), (10), (13), we then deduce that

$$
\begin{aligned}
\left|K_{z}(x, y)\right| \leq & \left|F_{\lambda, \tau}(z)\right| \frac{1}{V\left(x, \lambda^{1 / m}\right)}|f(z, \lambda)|\left|g\left(d^{m}(x, y) z^{-1}, \tau\right)\right| \\
\leq & C_{5} \frac{1}{(\cos \psi)^{n / m+\kappa}} \frac{1}{V\left(x,[\Re(z)]^{1 / m}\right)}\left[b\left(d^{m}(x, y) \Re\left(z^{-1}\right)\right)\right]^{1-\epsilon} \\
& \times|f(z, \Re(z))|\left|g\left(d^{m}(x, y) z^{-1}, d^{m}(x, y) \Re\left(z^{-1}\right)\right)\right| \\
\leq & C_{5} \frac{(\cos \arg z)^{n / m+\kappa}}{(\cos \psi)^{n / m+\kappa}} \frac{1}{V\left(x,[\Re(z)]^{1 / m}\right)}\left[b\left(d^{m}(x, y) \Re\left(z^{-1}\right)\right)\right]^{1-\epsilon},
\end{aligned}
$$

which completes the proof of Theorem 1 .

Applying Theorem 1 we can obtain the time derivatives estimate for heat kernels as follows.

Theorem 2. Under the same assumptions as in Theorem 1, there exists a constant $C_{7}>0$ such that for all $x, y \in \mathcal{X}, \epsilon \in(0,1), \psi \in(0, \phi)$ with $\sin \psi \leq 1 / 3, k \in \mathbb{N}$, and $t \in(0, \infty)$,

$$
\left|\frac{\partial^{k}}{\partial t^{k}} K_{t}(x, y)\right| \leq C_{7} \frac{k !}{[t \sin (\epsilon \psi)]^{k}} \frac{1}{V\left(x, t^{1 / m}\right)}\left[b\left(d^{m}(x, y) t^{-1}\right)\right]^{1-\epsilon} .
$$


Proof. Let $\psi \in(0, \phi)$ such that $\sin \psi \leq 1 / 3$. Then $2 \sin (\epsilon \psi) \leq 1-\sin (\epsilon \psi)$ and

$$
\cos (\epsilon \psi) \geq \sqrt{6} / 3>1 / 2
$$

for all $\epsilon \in(0,1)$. Let $\gamma$ be a circle centered at $t>0$ with radius $t \sin (\epsilon \psi)$. Then for all $z \in \gamma$, we have

$$
\begin{aligned}
\left|d^{m}(x, y) \Re\left(z^{-1}\right)-d^{m}(x, y) t^{-1}\right| & \leq\left|d^{m}(x, y) z^{-1}-d^{m}(x, y) t^{-1}\right| \\
& \leq\left|\frac{d^{m}(x, y)(z-t)}{z t}\right| \\
& \leq \frac{\sin (\epsilon \psi)}{1-\sin (\epsilon \psi)} d^{m}(x, y) t^{-1} \\
& \leq \frac{1}{2} d^{m}(x, y) t^{-1}
\end{aligned}
$$

which together with the fact that for all $s, t>0$ satisfying $|s-t| \leq t / 2$,

$$
\begin{aligned}
g(s, t) & \leq C_{3}\left(\frac{1+t}{1+s}\right)^{\kappa} \exp \left(\frac{1+s}{1+t}\right) \\
& =C_{3}\left(1-\frac{s-t}{1+s}\right)^{\kappa} \exp \left(1-\frac{s-t}{1+t}\right) \\
& \leq C_{3} 2^{\kappa} e^{3 / 2}
\end{aligned}
$$

gives that for all $z \in \gamma$,

$$
g\left(d^{m}(x, y) \Re\left(z^{-1}\right), d^{m}(x, y) t^{-1}\right) \leq C_{3} 2^{\kappa} e^{3 / 2} .
$$

From this, (2), the Cauchy theorem and Theorem 1 it follows that

$$
\begin{aligned}
\left|\frac{\partial^{k}}{\partial t^{k}} K_{t}(x, y)\right|= & \left|\int_{\gamma} \frac{k !}{2 \pi i} \frac{K_{z}(x, y)}{(t-z)^{k+1}} d z\right| \\
\leq & \int_{\gamma} \frac{k !}{2 \pi} \frac{C_{5} 2^{n / m+\kappa}}{[t \sin (\epsilon \psi)]^{k+1}} \frac{1}{V\left(x,[\Re(z)]^{1 / m}\right)}\left[b\left(d^{m}(x, y) \Re\left(z^{-1}\right)\right)\right]^{1-\epsilon}|d z| \\
\leq & C_{5} 2^{n / m+\kappa} \frac{k !}{2 \pi} \int_{\gamma} g\left(d^{m}(x, y) \Re\left(z^{-1}\right), d^{m}(x, y) t^{-1}\right)^{1-\epsilon}|d z| \\
& \times \frac{1}{[t \sin (\epsilon \psi)]^{k+1}} \frac{1}{V\left(x,(2 t / 3)^{1 / m}\right)}\left[b\left(d^{m}(x, y) t^{-1}\right)\right]^{1-\epsilon} \\
\leq & C_{3} C_{5} 2^{\kappa} 3^{n / m} e^{3 / 2} \frac{k !}{[t \sin (\epsilon \psi)]^{k}} \frac{1}{V\left(x, t^{1 / m}\right)}\left[b\left(d^{m}(x, y) t^{-1}\right)\right]^{1-\epsilon},
\end{aligned}
$$

which together with $C_{7}=C_{3} C_{5} 2^{\kappa} 3^{n / m} e^{3 / 2}$ completes the proof of Theorem 2 ,

Remark 1. (i) If we replace $V\left(x,[\Re(z)]^{1 / m}\right)$ in (4) and $V\left(x, t^{1 / m}\right)$ in (6), respectively, by $\left[V\left(x,[\Re(z)]^{1 / m}\right) V\left(y,[\Re(z)]^{1 / m}\right)\right]^{1 / 2}$ and $\left[V\left(x, t^{1 / m}\right) V\left(y, t^{1 / m}\right)\right]^{1 / 2}$, then the conclusions of Theorem 1 and Theorem 2 still hold with $V\left(x,[\Re(z)]^{1 / m}\right)$ in (7) and $V\left(x, t^{1 / m}\right)$ in (14) replaced by $\left[V\left(x,[\Re(z)]^{1 / m}\right) V\left(y,[\Re(z)]^{1 / m}\right)\right]^{1 / 2}$ and

$$
\left[V\left(x, t^{1 / m}\right) V\left(y, t^{1 / m}\right)\right]^{1 / 2},
$$

respectively.

(ii) If $K_{z}$ is holomorphic in $\Sigma_{\phi^{\prime}}$ with $\phi^{\prime} \in(0, \pi / 2]$ and $K_{t}$ satisfies (6) for $t \in$ $(0, \infty)$, arguing as in [10, 5], one can verify that (4) holds for $z \in \Sigma_{\phi}$ for each $\phi \in\left(0, \phi^{\prime}\right)$. Moreover, if $K_{t}$ is the kernel of semigroup $\left\{e^{-t \mathcal{L}}\right\}_{t>0}$, where $\mathcal{L}$ is a 
non-negative and self-adjoint operator on $L^{2}(\mathcal{X})$, and satisfies (6), then Lemma 2 in [8] indicates that $K_{t}$ is holomorphically continued to $z \in \Sigma_{\pi / 2}$ and (4) holds for all $z \in \Sigma_{\pi / 2}$.

(iii) The condition (3) is crucial in the proof of Theorem 1. A typical example which satisfies $(3)$ is that $b(s)=(1+s)^{-\kappa}$. Moreover, if $\widetilde{b}$ is a bounded decreasing function on $(0, \infty)$ and satisfies that there exists $\widetilde{\delta}>0$ such that

$$
\lim _{r \rightarrow \infty} \tilde{b}(r) r^{n+\widetilde{\delta}}=0
$$

where $n$ is as in (1), then by taking $b(r)=C(1+s)^{-(n+\gamma)}$ with $\gamma<\delta$, we have $\widetilde{b}(r) \leq b(r)$ for all $r>0$, and

$$
\lim _{r \rightarrow \infty} b(r)^{1-\epsilon} r^{n+\delta}=0
$$

for any $0<\epsilon<\gamma /(n+\gamma)$ and $\delta=\gamma(1-\epsilon)-n \epsilon$, which indicates that $b$ and $\widetilde{b}$ have a similar decay. Applying Theorem 1 and Theorem 2 for such $K_{z}$ and $b$, one obtains Proposition 3.3 in [10]. Moreover, these results indicate that the estimates on $z \in \Sigma_{\epsilon \psi}$ with $\psi \in(0, \phi)$ and the time derivative estimate for the heat kernel have a similar decay to the heat kernel $K_{t}$ itself, which is useful in applications; see [5, 10, 11]. 8].

The following proposition was essentially proved in the proof of Theorem 4 in

Proposition 1. Let $z \in \Sigma_{\pi / 2} \rightarrow K_{z} \in \mathbb{C}$ be the kernel of a holomorphic family of bounded operators on $L^{2}(\mathcal{X})$. Suppose that $0<\delta<1,0<\epsilon<1 / 8, x, y \in \mathcal{X}$ and $t>0$. Let $a, c$ be positive constants which may depend on $t, x, y, \epsilon$ and $\delta$ such that $0<c \leq 1,\left|K_{z}(x, y)\right| \leq a$,

$$
\begin{gathered}
\left|K_{s}(x, y)\right| \leq a c \\
\text { for }(1-\delta) t \leq s \leq(1+\delta) t, \text { and } z \in \Sigma_{\phi} \text { with } \Re(z) \geq(1-\delta) t \text {. Then } \\
\left|K_{t+i v}(x, y)\right| \leq a c^{1-3 \epsilon}
\end{gathered}
$$

for all $|v| \leq \epsilon \delta t$, and moreover, for all $k \in \mathbb{N}$,

$$
\left|\frac{\partial^{k}}{\partial t^{k}} K_{t}(x, y)\right| \leq \frac{k !}{(\epsilon \delta t)^{k}} a c^{1-3 \epsilon}
$$

From Proposition 1, it is easy to deduce the following conclusion.

Corollary 1. Let all the assumptions be the same as in Theorem 1 with $\phi=\pi / 2$. Suppose that $0<\delta<1$ and $0<\epsilon<1 / 8$. Then there exists a constant $C_{8}>0$ such that for all $x, y \in \mathcal{X}$ and $z \in \Sigma_{\psi}$ with $\tan \psi=\epsilon \delta$,

$$
\left|K_{z}(x, y)\right| \leq C_{8} \frac{1}{V\left(x,[\Re(z)]^{1 / m}\right)}\left[b\left(d^{m}(x, y)[\Re(z)]^{-1}\right)\right]^{1-3 \epsilon},
$$

and for all $k \in \mathbb{N}$ and $t \in(0, \infty)$,

$$
\left|\frac{\partial^{k}}{\partial t^{k}} K_{t}(x, y)\right| \leq C_{8} \frac{k !}{(t \epsilon \delta)^{k}} \frac{1}{V\left(x, t^{1 / m}\right)}\left[b\left(d^{m}(x, y) t^{-1}\right)\right]^{1-3 \epsilon} .
$$

Proof. For any $x, y \in \mathcal{X}$ and $t>0$, let

$$
a=C_{3} \frac{1}{V\left(x,[(1-\delta) t]^{1 / m}\right)}
$$


and $c=C_{7}^{-1} b\left(d^{m}(x, y) t^{-1}\right)$. Then $\left|K_{z}(x, y)\right| \leq a$ for all $\Re(z)>(1-\delta) t, 0<c<1$, and $\left|K_{s}(x, y)\right| \leq a c$ for all $(1-\delta) t \leq s \leq(1+\delta) t$. Applying Proposition 1 and the doubling condition (1) yields that for all $|v| \leq \epsilon \delta t$,

$$
\begin{aligned}
\left|K_{t+i v}(x, y)\right| & \leq a c^{1-3 \epsilon} \\
& \leq C_{3} \frac{1}{V\left(x,[(1-\delta) t]^{1 / m}\right)}\left[b\left(d^{m}(x, y) t^{-1}\right)\right]^{1-3 \epsilon} \\
& \leq C_{3} 2^{n / m} \frac{1}{V\left(x, t^{1 / m}\right)}\left[b\left(d^{m}(x, y) t^{-1}\right)\right]^{1-3 \epsilon},
\end{aligned}
$$

which indicates that for all $z \in \Sigma_{\psi}$ with $\tan \psi=\epsilon \delta$,

$$
\left|K_{z}(x, y)\right| \leq C_{3} 2^{n / m} \frac{1}{V\left(x,[\Re(z)]^{1 / m}\right)}\left[b\left(d^{m}(x, y)[\Re(z)]^{-1}\right)\right]^{1-3 \epsilon} .
$$

Similarly, for all $k \in \mathbb{N}$, we have

$$
\frac{\partial^{k}}{\partial t^{k}} K_{t}(x, y) \leq \frac{k !}{(\epsilon \delta t)^{k}} a c^{1-3 \epsilon} \leq C_{3} 2^{n / m} \frac{k !}{(\epsilon \delta t)^{k}} \frac{1}{V\left(x, t^{1 / m}\right)}\left[b\left(d^{m}(x, y) t^{-1}\right)\right]^{1-3 \epsilon},
$$

which together with $C_{8}=C_{3} 2^{n / m}$ completes the proof of Corollary 1 .

Remark 2. We remark that by Theorem 1, we can obtain the estimates for the heat kernel on $z \in \Sigma_{\phi}$ with any $\phi \in(0, \pi / 2]$. However, by Corollary 1, we can only obtain the estimates on $z \in \Sigma_{\psi}$ with $\psi=\arctan 1 / 8$.

\section{ACKNOWLEDGMENTS}

Dachun Yang would like to thank Professor Xuan Thinh Duong and Professor Lixin Yan for some constructive discussions on this topic.

\section{REFERENCES}

1. W. Arendt, Gaussian estimates and interpolation of the spectrum in $L^{p}$, Differential Integral Equations 7 (1994), 1153-1168. MR.1269649 (95e:47066)

2. P. Auscher, X. T. Duong and A. McIntosh, Boundedness of Banach space valued singular integral operators and applications to Hardy spaces, preprint, 2004.

3. G. Carron, T. Coulhon and E. M. Ouhabaz, Gaussian estimates and $L^{p}$-boundedness of Riesz means, J. Evol. Equ. 2 (2002), 299-317. MR1930609(2003i:35116)

4. R. R. Coifman and G. Weiss, Analyse harmonique non-commutative sur certains espaces homogènes, Lecture Notes in Math. 242, Springer-Verlag, Berlin, 1971. MR0499948 (58:17690)

5. T. Coulhon and X. T. Duong, Maximal regularity and kernel bounds: observations on a theorem by Hieber and Prüss, Adv. Differential Equations 5 (2000), 343-368. MR.1734546 (2001d:34087)

6. E. B. Davies, Heat Kernels and Spectral Theory, Cambridge Tracts in Mathematics, 92, Cambridge University Press, Cambridge, 1990. MR.1103113(92a:35035)

7. E. B. Davies, $L^{p}$ spectral independence and $L^{1}$ analyticity, J. London Math. Soc. (2) 52 (1995), 177-184. MR.1345724(96e:47034)

8. E. B. Davies, Non-Gaussian aspects of heat kernel behaviour, J. London Math. Soc. (2) 55 (1997), 105-125. MR 1423289(97i:58169)

9. X. T. Duong and E. M. Ouhabaz, Gaussian upper bounds for heat kernels of a class of nondivergence operators, International Conference on Harmonic Analysis and Related Topics, Sydney, 2002, Proc. Centre Math. Appl. Austral. Nat. Univ., Vol. 41, Austral. Nat. Univ., Canberra, 2003, pp. 35-45. MR1994513 (2004g:35104)

10. X. T. Duong and D. W. Robinson, Semigroup kernels, Poisson bounds, and holomorphic functional calculus, J. Funct. Anal. 142 (1996), 89-128. MR 1419418 (97j:47056)

11. X. T. Duong and L. Yan, Duality of Hardy and BMO spaces associated with operators with heat kernel bounds, J. Amer. Math. Soc. 18 (2005), 943-973. MR2163867 (2006d:42037) 
12. X. T. Duong and L. Yan, New function spaces of BMO type, the John-Nirenberg inequality, interpolation, and applications, Comm. Pure Appl. Math. 58 (2005), 1375-1420. MR 2162784 (2006i:26012)

13. E. M. Ouhabaz, Gaussian estimates and holomorphy of semigroups, Proc. Amer. Math. Soc. 123 (1995), 1465-1474. MR 1232142 (95f:47068)

14. E. M. Ouhabaz, Analysis of heat equations on domains, London Mathematical Society Monographs Series, 31, Princeton University Press, Princeton, NJ, 2005. MR2124040 (2005m:35001)

School of Mathematical Sciences, Beijing Normal University, Laboratory of Mathematics and Complex Systems, Ministry of Education, Beijing 100875, People's Republic OF CHINA

E-mail address: dcyang@bnu.edu.cn

School of Mathematical Sciences, Beijing Normal University, Laboratory of Mathematics and Complex Systems, Ministry of Education, Beijing 100875, People's Republic OF CHINA

E-mail address: yuanzhou@mail.bnu.edu.cn 